\title{
On a Singular Cauchy Problem for Functional Differential Equations with Non-Increasing Non-Linearities*
}

\author{
By \\ V. Pylypenko and A. Rontó \\ (National University of Ukraine, Ukraine and Academy of Sciences \\ of the Czech Republic, Czech Republic)
}

\begin{abstract}
We obtain general conditions sufficient for the solvability of a singular Cauchy problem for functional differential equations with non-increasing nonlinearities.

Key Words and Phrases. Functional differential problem, Singular Cauchy problem, Unbounded solution.

2000 Mathematics Subject Classification Numbers. 34K05, 34K10.
\end{abstract}

\section{Problem setting and introduction}

The aim of this note is to establish some general conditions sufficient for the existence of a solution of a singular Cauchy problem for a class of nonlinear functional differential equations. More precisely, we consider the equation

$$
u^{\prime}(t)=(g u)(t), \quad t \in(a, b],
$$

where $-\infty<a<b<\infty$ and $g: C((a, b], \boldsymbol{R}) \rightarrow L_{1 ; \text { loc }}((a, b], \boldsymbol{R})$ is a certain (generally speaking, non-linear) mapping which is assumed to be non-increasing with respect to the natural pointwise ordering (see Definition 4). Solutions of equation (1) are sought for in the class of locally absolutely continuous functions and, in particular, may be unbounded in a neigbourhood of the point $a$ (the precise notion of a solution is given by Definition 2 below).

Definition 1. One says that a function $u:(a, b] \rightarrow \boldsymbol{R}$ is locally absolutely continuous if its restriction $\left.u\right|_{[a+\varepsilon, b]}$ to the interval $[a+\varepsilon, b]$ is absolutely continuous for any $\varepsilon \in(0, b-a)$.

We are interested in conditions under which equation (1) has solutions satisfying the additional condition

$$
\lim _{t \rightarrow a+} h(t) u(t)=0
$$

\footnotetext{
* Research supported in part by AS CR, Institutional Research Plan No. AV0Z10190503.
} 
where $h:(a, b] \rightarrow \boldsymbol{R}$ is a given continuous function such that

$$
\lim _{t \rightarrow a+} h(t)=0
$$

and

$$
h(t)>0 \quad \text { for all } t \in(a, b] .
$$

Definition 2. By a solution of equation (1), we mean a locally absolutely continuous function $u:(a, b] \rightarrow \boldsymbol{R}$ which possesses the property $h u^{\prime} \in L_{1}((a, b], \boldsymbol{R})$ and satisfies equality (1) almost everywhere on the interval $(a, b]$.

It is important to point out that the derivative of a locally absolutely continuous function satisfying equality (1) may have a non-integrable singularity in a neighbourhood of the point $a$. For example, the function

$$
u(t)=\frac{\lambda}{t^{4}}, \quad t \in(0,1]
$$

for any real $\lambda$ satisfies the equality

$$
u^{\prime}(t)=-\frac{4}{t^{3}} u(\sqrt{t}), \quad t \in(0,1]
$$

where the coefficient function $(0,1] \ni t \mapsto-4 t^{-3}$ is non-integrable. The presence of a certain weight function is thus expected when dealing with the problems of this type. As such a weight function, our definition of a solution of equation (1) uses the same function $h$ with properties (3) and (4) that appears in condition (3). For example, function (5) turns out to be a solution of equation (6) if $h(t):=t^{\gamma}, t \in(0,1]$, with $\gamma>4$.

For the sake of simplicity, we assume throughout the paper that the above mentioned function $h$ has the following properties:

$$
\begin{gathered}
\text { The function } h:(a, b] \rightarrow(0,+\infty) \text { is non-decreasing } \\
\text { and possesses property (3). }
\end{gathered}
$$

These technical assumptions are not very restrictive because only the qualitative behaviour of $h$ in a neighbourhood of the point $a$ has influence on the formulation of the problems below and, thus, $h$ can be redefined in an arbitrary suitable manner in an neighbourhood of $b$. The function

$$
h(t)=(t-a)^{\gamma}, \quad t \in(a, b],
$$

where $\gamma \in(0,+\infty)$, is a typical example of a function satisifying conditions (7).

Definition 3. By a solution of problem (1), (2), a solution of equation (1) possessing property (2) is meant. 
We emphasize that the definition of a solution of the functional differential equation (1) is constructed here so that it involves the same function $h$ that determines the additional condition (2), i.e., Definition 2 in each case is adjusted to condition (2). For example, one can check directly that function (5) for arbitrary $\lambda \in \boldsymbol{R}$ and $\varepsilon \in(0,+\infty)$ is a solution of the problem

$$
\lim _{t \in(0,1]} t^{4+\varepsilon} u(t)=0
$$

for equation (6) in the sense of the definition above.

It should be noted that the property of the uniqueness of a solution is not typical for the problems of the class indicated. For example, the homogeneous problem (6), (8), as we have already seen above, has infinitely many solutions if $\varepsilon$ is positive.

Problem (1), (2) with $h$ satisfying (3) is usually referred to as the singular Cauchy problem (see, e.g., [6]). It reduces, in a natural way, to the classical regular Cauchy problem if $h$ is equal identically to a non-zero constant. Regular and singular Cauchy problems for various classes of functional differential equations are treated, in particular, in [2-4, 6, 11-15]. A problem on regular solutions possessing properties of type (2) is studied in [1].

\section{Notation}

The following notation is used throughout the paper.

1. $\boldsymbol{R}:=(-\infty, \infty), \boldsymbol{N}:=\{1,2,3, \ldots\}$.

2. If $-\infty<a<b<\infty$ and $A \subseteq[a, b]$ is a measurable set, then $L_{1}(A, \boldsymbol{R})$ is the Banach space of all the Lebesgue integrable functions $u: A \rightarrow \boldsymbol{R}$ with the standard norm

$$
L_{1}(A, \boldsymbol{R}) \ni u \mapsto \int_{A}|u(t)| d t
$$

3. $L_{1 ; \operatorname{loc}}((a, b], \boldsymbol{R})$ is the set of functions $u:(a, b] \rightarrow \boldsymbol{R}$ such that $\left.u\right|_{[a+\varepsilon, b]} \in L_{1}([a+\varepsilon, b], \boldsymbol{R})$ for any $\varepsilon \in(0, b-a)$.

4. $C((a, b], \boldsymbol{R})$ is the linear manifold of all the continuous functions $u:(a, b] \rightarrow \boldsymbol{R}$.

5. $A C_{\mathrm{loc}}((a, b], \boldsymbol{R})$ is the set of locally absolutely continuous functions $u:(a, b] \rightarrow \boldsymbol{R}$.

6. $A C_{\mathrm{loc} ; h}((a, b], \boldsymbol{R})$ is the set of all the locally absolutely continuous functions $u:(a, b] \rightarrow \boldsymbol{R}$ such that $h u^{\prime} \in L_{1}((a, b], \boldsymbol{R})$ and

$$
\sup _{t \in(a, b]} h(t)|u(t)|<+\infty .
$$




\section{General theorem}

Let us consider the functional differential equation

$$
u^{\prime}(t)=(g u)(t), \quad t \in(a, b],
$$

where $g: C((a, b], \boldsymbol{R}) \rightarrow L_{1 ; \operatorname{loc}}((a, b], \boldsymbol{R})$ is a certain mapping. Our main object here is the problem on finding solutions of equation (10) possessing property (2).

Definition 4. An operator $g: C((a, b], \boldsymbol{R}) \rightarrow L_{1 ; \operatorname{loc}}((a, b], \boldsymbol{R})$ is said to be non-increasing if the condition

$$
\left(g u_{1}\right)(t) \leq\left(g u_{0}\right)(t), \quad t \in(a, b],
$$

is satisfied for arbitrary pairs of functions $\left\{u_{0}, u_{1}\right\} \subset C((a, b], \boldsymbol{R})$ such that

$$
u_{1}(t) \geq u_{0}(t), \quad t \in(a, b]
$$

The standard Definition 4 here describes the class of non-linear terms that may appear at the right-hand side of equation (10) under conditions of the theorems formulated below.

Definition 5. We say that a locally absolutely continuous function $u:(a, b] \rightarrow \boldsymbol{R}$ belongs to the set $A C_{\mathrm{loc} ; h}((a, b], \boldsymbol{R})$ if $h u^{\prime} \in L_{1}((a, b], \boldsymbol{R})$ and relation (9) is satisfied.

The theorem given below provides general conditions guaranteeing the existence of solutions satisfying the condition

$$
\lim _{t \rightarrow a+} h(t) u(t)=0,
$$

that is, the singular Cauchy problem (10), (11) is considered.

Theorem 1. Let the mapping $g: C((a, b], \boldsymbol{R}) \rightarrow L_{1 ; 1 \text { loc }}((a, b], \boldsymbol{R})$ in equation (10) be non-increasing and, moreover, the conditions

$$
h g\left(\frac{\lambda}{h}\right) \in L_{1}((a, b], \boldsymbol{R})
$$

and

$$
\lim _{t \rightarrow a+} h(t) \int_{t}^{b} g\left(\frac{\lambda}{h}\right)(s) d s=0
$$

be satisfied for any $\lambda \in \boldsymbol{R}$. Furthermore, let there exist certain functions $\psi_{0}$ and $\psi_{1}$ in $A C_{\mathrm{loc} ; h}((a, b], \boldsymbol{R})$ such that

$$
\lim _{t \rightarrow a+} h(t) \psi_{i}(t)=0, \quad i=0,1,
$$


and the inequalities

$$
(-1)^{i}\left(\psi_{1}^{(i)}(t)-\psi_{0}^{(i)}(t)\right) \geq 0, \quad t \in(a, b], i=0,1,
$$

and

$$
(-1)^{i}\left(\psi_{i}^{\prime}(t)-\left(g \psi_{i}\right)(t)\right) \geq 0, \quad t \in(a, b], i=0,1,
$$

are satisfied.

Then equation (10) has a solution $u:(a, b] \rightarrow \boldsymbol{R}$ possessing property (11) and, moreover, the estimates

$$
\psi_{0}(t) \leq u(t) \leq \psi_{1}(t), \quad t \in(a, b],
$$

and

$$
\psi_{0}^{\prime}(t) \geq u^{\prime}(t) \geq \psi_{1}^{\prime}(t), \quad t \in(a, b]
$$

are true.

Note that, due to (7), the function $\lambda / h$ is continuous on $(a, b]$ and, therefore, the function $g(\lambda / h)$ appearing in relations (12) and (13) is well defined.

Remark 1. For $g$ homogeneous, it is sufficient to suppose that

$$
h g\left(\frac{1}{h}\right) \in L_{1}((a, b], \boldsymbol{R})
$$

because (19) guarantees the fulfilment of (12) for any $\lambda$ in that case. In the general case, however, condition (19) may not imply the property indicated. Indeed, let us take $a=0, b=1$, fix a certain $\varepsilon \in(0,1)$, and, for any nonnegative $u$ from $C((0,1], \boldsymbol{R})$, put

$$
(g u)(t):=-\frac{1}{t^{m_{u}} h(t)}, \quad t \in(0,1],
$$

where

$$
m_{u}:= \begin{cases}1-\varepsilon & \text { if } \mu_{u} \leq 1 \\ 1 & \text { if } \mu_{u}>1\end{cases}
$$

and $\mu_{u}:=\sup _{t \in(a, b]} h(t) u(t)$. For all other functions $u$ from $C((0,1], \boldsymbol{R})$, we put $g u:=0$. Then, as can be checked, the operator $g: C((a, b], \boldsymbol{R}) \rightarrow L_{1 ; \operatorname{loc}}((a, b], \boldsymbol{R})$ given by equality (20) is non-increasing in the sense of Definition 4 .

Since $m_{1 / h}=1-\varepsilon$, relation (12) is satisfied for $\lambda \leq 1$ (and, in particular, (19) is true). However, relation (12) does not hold if $\lambda>1$. 
Remark 2. The solution of problem (10), (11), the existence of which is stated in Theorem 1, can be found approximately by using a convergent monotone two-sided iteration procedure.

\section{Corollaries for linear equations}

Let us formulate corollaries for the case where the functional differential equation (10) has the form

$$
u^{\prime}(t)=(l u)(t)+q(t), \quad t \in(a, b]
$$

where $l: C((a, b], \boldsymbol{R}) \rightarrow L_{1 ; \operatorname{loc}}((a, b], \boldsymbol{R})$ is a certain linear mapping and $q$ is a given function from $L_{1 ; \operatorname{loc}}((a, b], \boldsymbol{R})$.

Definition 6. An operator $l: C((a, b], \boldsymbol{R}) \rightarrow L_{1 ; \operatorname{loc}}((a, b], \boldsymbol{R})$ is said to be negative if the inequality $(l u)(t) \leq 0$ is satisfied for a.e. $t \in(a, b]$ whenever $u \in C((a, b], \boldsymbol{R})$ is such that

$$
\inf _{t \in(a, b]} u(t) \geq 0 .
$$

Definition 7. Let $u$ and $v$ be some functions from $A C_{\mathrm{loc}}((a, b], \boldsymbol{R})$. We say that a function $q:(a, b] \rightarrow \boldsymbol{R}$ belongs to the set $M_{l}(u, v)$ if it satisfies the estimate

$$
v^{\prime}(t)-(l v)(t) \leq q(t) \leq u^{\prime}(t)-(l u)(t)
$$

for a.e. $t \in(a, b]$.

The following statement on problem (21), (11) is true.

Corollary 1. Let the mapping $l$ in (21) be negative, the condition

$$
h l\left(\frac{1}{h}\right) \in L_{1}((a, b], \boldsymbol{R})
$$

be satisfied, and, moreover,

$$
\lim _{t \rightarrow a+} h(t) \int_{t}^{b} l\left(\frac{1}{h}\right)(s) d s=0 .
$$

Furthermore, let the function $q \in L_{1 ; \operatorname{loc}}((a, b], \boldsymbol{R})$ be such that

$$
h q \in L_{1}((a, b], \boldsymbol{R}), \quad \lim _{t \rightarrow a+} h(t) \int_{t}^{b} q(s) d s=0
$$

and $q \in M_{l}\left(\psi_{0}, \psi_{1}\right)$, where $\psi_{0}$ and $\psi_{1}$ are certain functions from $A C_{\mathrm{loc} ; h}((a, b], \boldsymbol{R})$ satisfying inequalities (14), (15), (16). 
Then equation (21) has a solution $u:(a, b] \rightarrow \boldsymbol{R}$ possessing property (11) and satisfying estimates (17) and (18).

Note that condition (16) is satisfied if and only if $0 \in M_{l}\left(\psi_{0}, \psi_{1}\right)$.

Corollary 2. Let the mapping $l$ in (21) be negative and such that relations (22) and (23) hold. Furthermore, assume that the function $q \in L_{1 ; \operatorname{loc}}((a, b], \boldsymbol{R})$ satisfies conditions (24) and, for some $i \in\{0,1\}$, the inequality

$$
(-1)^{i}\left(\psi^{\prime}(t)-(l \psi)(t)\right) \leq(-1)^{i} q(t) \leq 0, \quad t \in(a, b],
$$

holds with a certain function $\psi \in A C_{\mathrm{loc} ;}((a, b], \boldsymbol{R})$ such that

$$
\lim _{t \rightarrow a+} h(t) \psi(t)=0
$$

and

$$
(-1)^{i} \psi(t) \geq 0, \quad t \in(a, b] .
$$

Then equation (21) has a solution $u:(a, b] \rightarrow \boldsymbol{R}$ possessing property (11). Moreover, this solution and its derivative admit the estimates

$$
0 \leq(-1)^{i+k} u^{(k)}(t) \leq(-1)^{i+k} \psi^{(k)}(t), \quad t \in(a, b], i, k=0,1 .
$$

Note that the absence of extra assumptions on the functions $\psi_{0}$ and $\psi_{1}$ is partly explained by the unpleasant fact that the set $M_{l}\left(\psi_{0}, \psi_{1}\right)$ may turn out to be empty. This never happens, for example, if the mapping $-l$ is negative because, as can be verified, then

$$
M_{l}\left(\psi_{0}, \psi_{1}\right) \neq \varnothing
$$

for arbitrary functions $\psi_{0}$ and $\psi_{1}$ satisfying estimates (15). In our case, however, the operator $l$ is negative and, therefore, the property mentioned essentially depends on the choice of $\psi_{0}$ and $\psi_{1}$.

\section{Auxiliary statements and proofs}

In the sequel we need an abstract theorem on operators in partially ordered normed spaces [8, Theorem 38.2]. In order to state it, we first formulate definitions. We use the books $[7,8]$ as the main references (see also $[5,9]$ ).

\subsection{General notions}

Let $X$ be a normed space over $\boldsymbol{R}$ and $P$ be a cone [7] in $X$, i.e., a non-empty closed subset of $X$ possessing the properties $P \cap(-P)=\{0\}$ and 
$\alpha_{1} P+\alpha_{2} P \subseteq P$ for all $\left\{\alpha_{1}, \alpha_{2}\right\} \subset[0,+\infty)$. A cone $P$ generates a natural partial ordering of $X$. As usual, we shall write $u \leqq_{P} v$ and $v \geqq_{P} u$ if and only if $v-u \in P$. A set $\mathscr{M} \subset X$ is said to be order bounded from above (resp., below) if there exists some $w \in X$ such that $x \geqq_{P} w$ (resp., $x \geqq_{P} w$ ) for all $x \in \mathscr{M}$, and order bounded if it is so from above and from below simultaneously.

A sequence $\left\{u_{k} \mid k \geq 0\right\} \subset X$ is called non-decreasing if $u_{k} \leqq_{P} u_{k+1}$ for any $k \geq 0$. A cone $P \subset X$ is said to be regular if every non-decreasing sequence order bounded from above converges in $X$. As usual, we say that an operator $T: X \rightarrow X$ is non-decreasing (with respect to $P$ ) if $T u \geqq_{P} T v$ for any $u$ and $v$ from $X$ such that $u \geqq_{P} v$.

Definition 8 ([8]). An operator $T: X \rightarrow X$ is said to be limit-monotone compact on an order bounded set $\mathscr{M} \subset X$ if, for any sequence $\left\{u_{n}: n \geq 0\right\} \subset \mathscr{M}$ such that

$$
u_{0} \leqq_{P} T u_{1} \varliminf_{P} T^{2} u_{2} \leqq_{P} \cdots,
$$

the sequence $\left\{T^{n} u_{n}: n \geq 0\right\}$ converges.

Theorem 2 ([8, Theorem 38.2]). Let $T: X \rightarrow X$ be a non-decreasing operator which is limit-monotone compact on an order bounded closed set $\mathscr{M} \subset X$ and maps $\mathscr{M}$ into itself. Moreover, assume that the cone $P$ is regular and there exists some $\psi_{0} \in \mathscr{M}$ for which

$$
T \psi_{0} \geqq_{P} \psi_{0} .
$$

Then the operator $T$ has at least one fixed point in $\mathscr{M}$.

We assume that the function $h$ involved in the non-local condition (11) possesses properties (7).

Lemma 1. The set $A C_{\mathrm{loc} ; h}((a, b], \boldsymbol{R})$ is a Banach space with respect to the norm

$$
A C_{\mathrm{loc} ; h}((a, b], \boldsymbol{R}) \ni u \mapsto\|u\|:=\int_{a}^{b} h(s)\left|u^{\prime}(s)\right| d s+\sup _{\xi \in(a, b]} h(\xi)|u(\xi)| .
$$

Recall that the set $A C_{\mathrm{loc} ;}((a, b], \boldsymbol{R})$ is introduced by Definition 5 .

Lemma 2. The set

$$
K:=\left\{u \in A C_{\mathrm{loc} ; h}((a, b], \boldsymbol{R}): \inf _{t \in(a, b]} u(t) \geq 0 \text { and } \underset{t \in(a, b]}{\operatorname{ess} \sup } u^{\prime}(t) \leq 0\right\}
$$

is a regular cone in the space $A C_{\mathrm{loc} ; h}((a, b], \boldsymbol{R})$.

Lemmata 1 and 2 are proved in [10]. 
Lemma 3. If the mapping $g: C((a, b], \boldsymbol{R}) \rightarrow L_{1 ; \operatorname{loc}}((a, b], \boldsymbol{R})$ is nonincreasing, then, for any $u$ from $A C_{\mathrm{loc} ;}((a, b], \boldsymbol{R})$, there exists a certain $\mu_{u} \in[0,+\infty)$ such that

$$
g\left(\frac{\mu_{u}}{h}\right)(t) \leq(g u)(t) \leq g\left(\frac{-\mu_{u}}{h}\right)(t)
$$

for a.e. $t \in(a, b]$.

Proof. Let $u \in A C_{\mathrm{loc} ; h}((a, b], \boldsymbol{R})$ be an arbitrary function. Then

$$
\mu_{u}:=\sup _{t \in(a, b]} h(t)|u(t)|<+\infty,
$$

and, hence, the estimate

$$
|u(t)| \leq \frac{\mu_{u}}{h(t)}
$$

holds for any $t \in(a, b]$. Therefore,

$$
-\frac{\mu_{u}}{h(t)} \leq-|u(t)| \leq u(t) \leq|u(t)| \leq \frac{\mu_{u}}{h(t)}, \quad t \in(a, b] .
$$

By assumption, the mapping $g$ is non-increasing in the sense of Definition 4 . Therefore, relation (31) yields

$$
g\left(-\frac{\mu_{u}}{h}\right)(t) \geq g(-|u|)(t) \geq(g u)(t) \geq(g|u|)(t) \geq g\left(\frac{\mu_{u}}{h}\right)(t)
$$

for a.e. $t \in(a, b]$, whence estimate (30) follows immediately.

Lemma 4. The set

$$
\Pi_{0}(h):=\left\{u \in A C_{\mathrm{loc} ; h}((a, b], \boldsymbol{R}) \mid \lim _{t \rightarrow a+} h(t) u(t)=0\right\}
$$

is a closed linear subspace in $A C_{\mathrm{loc} ; h}((a, b], \boldsymbol{R})$.

Proof. Let $\left\{u_{m} \mid m \geq 1\right\} \subset \Pi_{0}(h)$ be an arbitrary sequence convergent, in terms of norm (28), to a certain element $u \in A C_{\mathrm{loc} ; h}((a, b], \boldsymbol{R})$. This means, in particular, that

$$
\lim _{m \rightarrow+\infty} \sup _{t \in(a, b]} h(t)\left|u_{m}(t)-u(t)\right|=0 .
$$

Let us fix an arbitrary positive $\varepsilon$. In view of (34), there exists a certain $m_{\varepsilon} \in \boldsymbol{N}$ such that

$$
h(t)\left|u_{m}(t)-u(t)\right| \leq \frac{\varepsilon}{2}
$$


for any $t \in(a, b]$ and $m \geq m_{\varepsilon}$. By assumption, $u_{m_{\varepsilon}} \in \Pi_{0}(h)$ and, therefore, there exists some $\delta_{\varepsilon}>0$ such that

$$
\left|u_{m_{\varepsilon}}(t)\right| h(t)<\frac{\varepsilon}{2}
$$

whenever $t \in(a, b]$ is such that $|t-a|<\delta_{\varepsilon}$. Combining (35) and (36), we find that

$$
|u(t)| h(t) \leq\left|u_{m_{\varepsilon}}(t)\right| h(t)+h(t)\left|u_{m}(t)-u(t)\right|<\varepsilon
$$

for $|t-a|<\delta_{\varepsilon}$. The arbitrariness of $\varepsilon$ now implies that $u \in \Pi_{0}(h)$, which, in view of the arbitrariness of the sequence $\left\{u_{m} \mid m \geq 1\right\}$, proves our lemma.

We set

$$
(T u)(t):=u(b)-\int_{t}^{b}(g u)(s) d s, \quad t \in(a, b],
$$

for any $u$ from $A C_{\mathrm{loc} ; h}((a, b], \boldsymbol{R})$.

Lemma 5. Assume that $g: C((a, b], \boldsymbol{R}) \rightarrow L_{1 ; \operatorname{loc}}((a, b], \boldsymbol{R})$ is non-increasing, the function $h$ possesses properties (7), and condition (12) holds. Then

1. $T$ is a well-defined mapping from $A C_{\mathrm{loc} ; h}((a, b], \boldsymbol{R})$ to $A C_{\mathrm{loc} ; h}((a, b], \boldsymbol{R})$;

2. $T: A C_{\mathrm{loc} ; h}((a, b], \boldsymbol{R}) \rightarrow A C_{\mathrm{loc} ; h}((a, b], \boldsymbol{R})$ is a mapping non-decreasing with respect to cone (29);

3. If condition (13) holds, then the inclusion

$$
T\left(\Pi_{0}(h)\right) \subseteq \Pi_{0}(h)
$$

is satisfied.

Proof. Assertions 1 and 2 are established similarly to the argument of [10], and we thus prove assertion 3 only.

Let $u \in \Pi_{0}(h)$ be arbitrary. Then, according to (33), $u$ posesses property (11) and, by virtue of Lemma 3 , one can specify a certain constant $\mu_{u} \in(0,+\infty)$ such that estimate $(30)$ is true. Then

$$
h(t) \int_{t}^{b} g\left(\frac{\mu_{u}}{h}\right)(s) d s \leq h(t) \int_{t}^{b}(g u)(s) d s \leq h(t) \int_{t}^{b} g\left(\frac{-\mu_{u}}{h}\right)(s) d s
$$

for a.e. $t$ from $(a, b]$. Estimate (40) and assumption (13) yield

$$
\lim _{t \rightarrow a+} h(t) \int_{t}^{b}(g u)(s) d s=0 .
$$


Taking relation (41) and definition (38) of the operator $T$ into account, we obtain

$$
\lim _{t \rightarrow a+} h(t)(T u)(t)=0,
$$

whence it follows that the function $T u$ belongs to the set $\Pi_{0}(h)$. The arbitrariness of $u$ from $\Pi_{0}(h)$ thus leads us to inclusion (39).

Lemma 6. A function $u:(a, b] \rightarrow \boldsymbol{R}$ from $A C_{\mathrm{loc} ;}((a, b], \boldsymbol{R})$ is a solution of problem (10), (11) if and only if it is a fixed point of mapping (38) lying in the set $\Pi_{0}(h)$.

This last lemma is an immediate consequence of relations (33), (38) and Definition 5 of the set $A C_{\mathrm{loc} ; h}((a, b], \boldsymbol{R})$.

\subsection{Proof of Theorem 1}

We are going to use Theorem 2. By Lemma 4,

$$
X:=\Pi_{0}(h)
$$

is a subspace of $A C_{\mathrm{loc} ; h}((a, b], \boldsymbol{R})$ and, hence, can be regarded as an independent Banach space with the same norm (28). Lemmata 1 and 2 guarantee that the set

$$
P:=K \cap \Pi_{0}(h)
$$

where $K$ is given by (29), is a regular cone in the Banach space $\Pi_{0}(h)$. By Lemma 5 , the operator $T: A C_{\mathrm{loc} ; h}((a, b], \boldsymbol{R}) \rightarrow A C_{\mathrm{loc} ; h}((a, b], \boldsymbol{R})$ defined by formula (38) has property (39) and, thus, we can pass to its restriction to $\Pi_{0}(h)$. The same Lemma 5 then ensures that $T: \Pi_{0}(h) \rightarrow \Pi_{0}(h)$ is non-decreasing with respect to the cone $P$.

Assumption (14) ensures that $\left\{\psi_{0}, \psi_{1}\right\} \subset \Pi_{0}(h)$, where $\psi_{0}$ and $\psi_{1}$ are the functions appearing in the formulation of the theorem. Furthermore,

$$
(-1)^{i}\left(\left(T \psi_{0}\right)^{(i)}(t)-\psi_{0}^{(i)}(t)\right) \geq 0, \quad t \in(a, b], i=0,1,
$$

and

$$
(-1)^{i}\left(\left(T \psi_{1}\right)^{(i)}(t)-\psi_{1}^{(i)}(t)\right) \leq 0, \quad t \in(a, b], i=0,1 .
$$

Indeed, it follows immediately from (16) and (38) that relations (44) and (45) are satisfied with $i=1$. Relations (44) and (45) with $i=0$ are obtained from the corresponding estimates with $i=1$ by integrating them from $t \in(a, b]$ to $b$. Hence, the relations (27) and

$$
T \psi_{1} \leqq_{P} \psi_{1}
$$

hold with respect to the cone $P$ given by formula (43). 
Since $T$ is non-decreasing, it follows from (27) and (46) that $T(\mathscr{M}) \subseteq \mathscr{M}$, where

$$
\mathscr{M}:=\left\{x \in \Pi_{0}(h): \psi_{0} \leqq_{K_{0}} x \leqq_{K_{0}} \psi_{1}\right\} .
$$

Since $\mathscr{M}$ is order bounded, it follows that $T: \Pi_{0}(h) \rightarrow \Pi_{0}(h)$ is limitmonotone compact on this set (see, e.g., [8]). Theorem 2 can now be used.

Applying Theorem 2 with $X$ and $P$ given by equalities (42) and (43) and using Lemma 6 , we obtain the existence of at least one solution $u$ of problem (10), (11) belonging to set (47) and, therefore, satisfying the estimate

$$
\psi_{0} \leqq_{P} u \leqq_{P} \psi_{1} .
$$

Finally, noticing that estimates (17) and (18) follow directly from (48), we complete the proof of Theorem 1 .

\subsection{Proofs of Corollaries 1 and 2}

In order to obtain Corollary 1 , it is sufficient to notice that, under assumptions (22) and (23), the mapping $g: C((a, b], \boldsymbol{R}) \rightarrow L_{1 ; \operatorname{loc}}((a, b], \boldsymbol{R})$ given by the formula

$$
(g u)(t):=(l u)(t)+q(t), \quad t \in(a, b],
$$

satisfies conditions (12) and (13) for any $\lambda$, by virtue of the linearity of $l$. Therefore, Theorem 1 can be applied. The assertion of Corollary 2 is an immediate consequence of Corollary 1 in the case where

$$
\psi_{k}:=((1-k) i+k(1-i)) \psi
$$

for $k=0,1$.

\section{Acknowledgement}

We are grateful to the anonymous referee for his careful reading the manuscript and valuable remarks.

\section{References}

[1] Agarwal, R. P. and Rontó, A., Linear functional differential equations possessing solutions with a given growth rate, J. Inequal. Appl., 2005 (2005), 49-65.

[2] Azbelev, N. V., Maksimov, V. P. and Rakhmatullina, L. F., Introduction to the theory of functional differential equations: methods and applications, Contemporary Mathematics and Its Applications, 3, Hindawi Publishing Corporation, Cairo, 2007.

[ 3 ] Dilna, N. Z. and Ronto, A. N., General conditions for the unique solvability of an initialvalue problem for nonlinear functional-differential equations, Ukrainian Math. J., 60 (2008), 191-198. 
